\title{
Utilidad de ePEAT en determinar candidatura para implante coclear en pacientes pediátricos con malformaciones de oído interno. Comunicación preliminar
}

\author{
Use of ePEAT to determine candidacy for cochlear implant in pediatric patients \\ with inner ear malformations. Preliminary communication
}

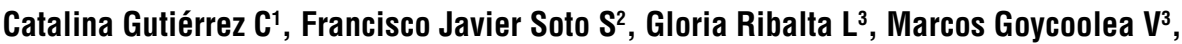 \\ Mauricio Cohen V3.
}

\begin{abstract}
RESUMEN
En candidatos a implante coclear con malformaciones del oído interno donde se encuentra un nervio coclear anormal, los estudios tradicionales y las imágenes muchas veces no pueden dar respuesta definitiva acerca de la funcionalidad y presencia del nervio coclear. Para esto ayudarían los estudios de electrofisiología.

Se presentan tres casos clínicos de pacientes con malformaciones del oído interno que fueron evaluados con ePEAT para ayudar a determinar su candidatura a implante coclear.

Los estudios electrofisiológicos no reemplazan a los estudios tradicionales de evaluación auditiva ni a los estudios por imágenes, sino que los complementan. Los casos presentados, demuestran que en casos de malformaciones de oído interno o CAl muy estrecho, en que se cuestiona seriamente la existencia de un nervio coclear funcional, y en casos de neuropatía auditiva, se hace necesario evaluar la función de la cóclea separadamente de la del nervio auditivo y la función del tronco. Para esto se utilizamos los ePEAT.

Los ePEAT entregan información valiosísima ya que nos permite conocer las reales capacidades de los pacientes para transmitir un estímulo auditivo hacia el sistema nervioso central, definiendo mejor las expectativas con el uso implante, asistiéndonos en nuestra toma de decisiones.

Palabras clave: ePEAT, implante coclear, malformaciones oído interno, hipoacusia sensorioneural, estudios electrofisiológicos.
\end{abstract}

\footnotetext{
ABSTRACT

In cochlear implant candidates with inner ear malformations, where there is an abnormal cochlear nerve, traditional studies and images cannot often provide definitive answers about the functionality and presence of the cochlear nerve. In these cases, electrophysiology studies can be used.

We present 3 cases of patients with inner ear malformations who were evaluated with ePEAT to determine their candidacy for a cochlear implant.

Médico General. Capacitación ORL en Clínica Las Condes.

Neurólogo, Clínica Las Condes.

Otorrinolaringólogo, Clínica Las Condes.
} 
Electrophysiological studies do not replace traditional hearing screening studies or imaging studies, but complement them. The cases presented in this study demonstrate that in patients with inner ear malformations or very narrow internal auditory canal, where we question the existence of a functional cochlear nerve, and in cases of auditory neuropathy, it is necessary to evaluate the cochlear function separately from the auditory nerve and from the brainstem. In these cases we use ePEAT.

ePEAT give us valuable information about the real abilities of patients to transmit an auditory stimulus to the central nervous system, which help us to define expectations with cochlear implant use, assisting us in our decision-making.

Key words: PPEAT, Cochlear Implant, Inner ear malformations, sensorineural hearing loss, Electrophysiological tests.

\section{INTRODUCCIÓN}

Dentro de la evaluación de los pacientes candidatos a implante coclear (IC) se encuentran los estudios auditivos y las imágenes. Las principales herramientas disponibles para un estudio auditivo objetivo son las Emisiones Otoacústicas (EOA), los Potenciales Evocados Auditivos de Tronco Cerebral (PEAT) y el reflejo acústico, y para el estudio subjetivo, la audiometría y pruebas de discriminación. En los pacientes con malformaciones del oído interno, este tipo de exámenes a veces no es suficiente para poder realizar una evaluación auditiva completa. En ocasiones, pacientes que presentan ausencia de respuesta en los exámenes tradicionales aún plantean dudas sobre si son o no candidatos a recibir un IC. Los estudios por imágenes entregan información adicional fundamental al evaluar a estos pacientes. Sin embargo, en malformaciones de oído interno en que se encuentra un nervio coclear anormal, las técnicas de imágenes no pueden muchas veces dar una respuesta definitiva a la pregunta de si el nervio coclear está presente dentro del conducto auditivo interno (CAI) pero hipoplásico, si es funcionante, 0 si se encuentra ausente. Normalmente en la resonancia nuclear magnética (RNM), el VIII par se observa más grueso y posterior al nervio facial. Si éste se observa del mismo diámetro o más fino que el facial, se considera hipoplásico ${ }^{1}$. Cuando el CAl se observa fino (menos de $3 \mathrm{~mm}$ de diámetro), también se debe sospechar una anomalía del VIII par'.

En todos estos casos lo adecuado es contar con estudios de electrofisiología para corroborar la conexión del nervio coclear al tronco ${ }^{2,3}$.
De la misma manera, los individuos con desórdenes en el espectro de la neuropatía auditiva (NA) también se beneficiarían del estudio electrofisiológico. Estos pacientes se caracterizan por presentar EOA positivas y PEAT ausentes 0 anormales.

Los pacientes con espectro de neuropatía auditiva, se componen de dos grupos, aquellos con daño real del nervio coclear (retrococlear), y aquellos con preservación de células ciliadas externas, daño de las células ciliadas internas y eventual nervio auditivo funcionante, lo que explicaría las EOA normales (por preservación de células ciliadas externas) y PEAT alterado (células ciliadas internas alteradas).

Los primeros corresponden aproximadamente a $1 / 4$ de los casos de NA, y son malos candidatos a IC, pues el nervio coclear no transmitirá la señal auditiva hacia el sistema nervioso central (SNC), en cambio la mayoría de los pacientes (3/4) corresponden al segundo grupo, y son excelentes candidatos a IC ${ }^{4,5}$.

Para poder diferenciar ambos grupos de pacientes se puede realizar PEAT con estímulo eléctrico (ePEAT) ya que este tipo de estímulo evalúa directamente la respuesta del nervio auditivo y del tronco para responder a estímulos eléctricos. El ePEAT imita el implante coclear antes de ser implantado. Estudios reportan que al implantar pacientes con neuropatía auditiva con ePEAT normales, estos tendrían mejores resultados en la evaluación del lenguaje que aquellos con ePEAT alterados, que serían aquellos sujetos con verdadero problema en el nervio coclear, e incluso que aquellos pacientes implantados por otras causas distintas a la neuropatía auditiva ${ }^{4,5}$.

El ePEAT se comenzó a aplicar para verificar la función neural de los pacientes candidatos a IC. 
Estimula con señales eléctricas, transmitidas por una aguja que se coloca con una técnica transtimpánica, la pared medial del oído medio (Figura 1). La obtención de los potenciales evocados del tronco cerebral es igual que cuando de utiliza estímulo acústico. Para realizar el examen, se requiere de anestesia general en niños ${ }^{6}$ (Figura 2) 0 anestesia local en adultos y miringotomía posterior (se debe ver la ventana redonda) para colocar el electrodo en el nicho de la ventana o lo más cercano a ésta (Figura 3). Hay discusión en la real importancia del sitio de estimulación. Los reportes de la literatura indican una tendencia hacia umbrales menores cuando el electrodo está ubicado lo más cercano posible a la ventana redonda ${ }^{7}$. El estímulo eléctrico es transferido por el electrodo para estimular cualquier fibra del nervio auditivo activa con el fin de provocar un potencial evocado de tronco, similar a como el IC se salta las células ciliadas internas para estimular al nervio auditivo.

A continuación se presentan tres casos clínicos de pacientes con malformaciones del oído interno que fueron evaluados con ePEAT para ayudar a determinar su candidatura a IC.

\section{CASOS CLÍNICOS}

\section{Caso 1}

Lactante menor de 2 años, sexo femenino. Con hipoacusia sensorioneural (HSN) profunda bilateral, EOA $(+)$, PEAT sin respuesta. Tomografía computarizada (TC) de oídos muestra una cóclea de aspecto normal y CAl estrecho (Figura 4). En la RNM se observa duplicación del CAI con un conducto filiforme para el VII par (Figura 5). No se ve claramente el nervio coclear pero la cóclea está desarrollada, por lo que se consideraría una mala candidata a IC. Para comprobar esta situación, se realizó ePEAT en el que no se observó respuesta, lo que la confirmó como mal candidato a IC (Figura 6).

\section{Caso 2}

Escolar masculino de 9 años de edad. Presenta un síndrome cráneo-facial complejo no caracterizado e HSN profunda bilateral sin respuesta a audífonos. EI TC de oídos muestra una otitis media con efusión (OME) crónica y una partición incompleta coclear

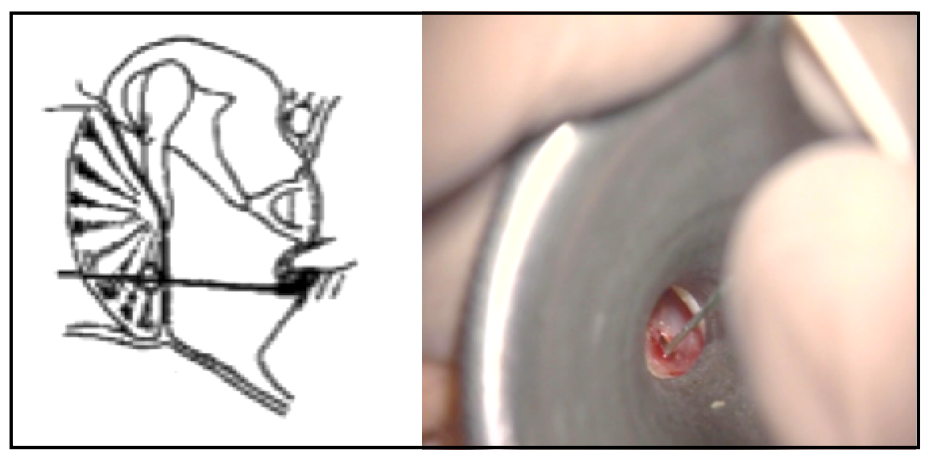

Figura 1. Colocación transtimpánica del electrodo.

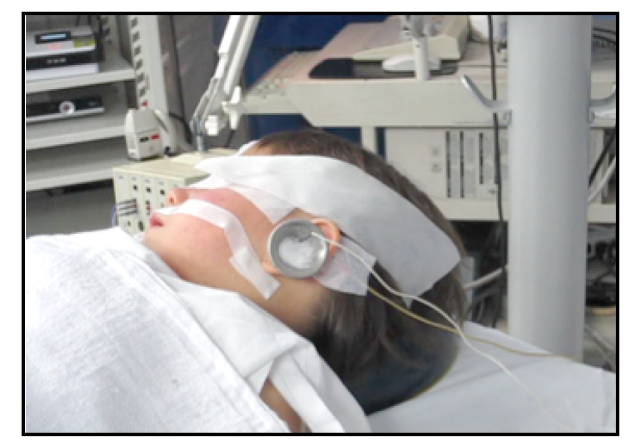

Figura 2. Técnica de fijación de electrodos para ePEAT.

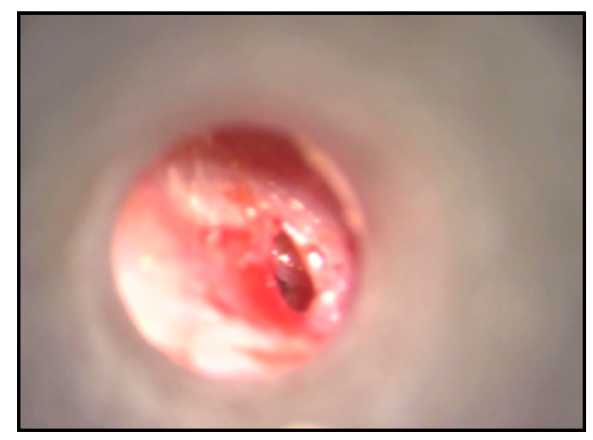

Figura 3. Miringotomía posterior. 


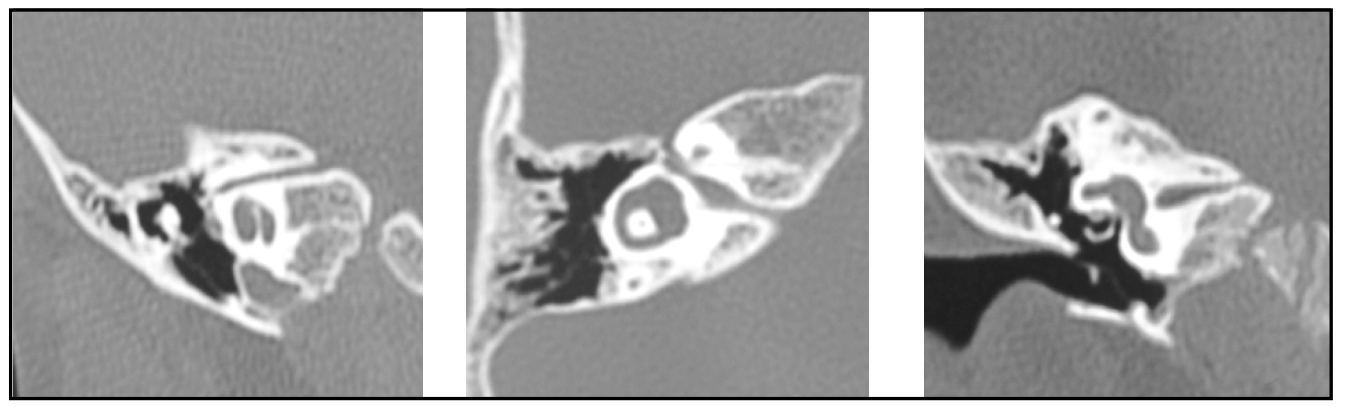

Figura 4. TC primer caso: CAI duplicado, con canal para VII par independiente, porción inferior estrecha.

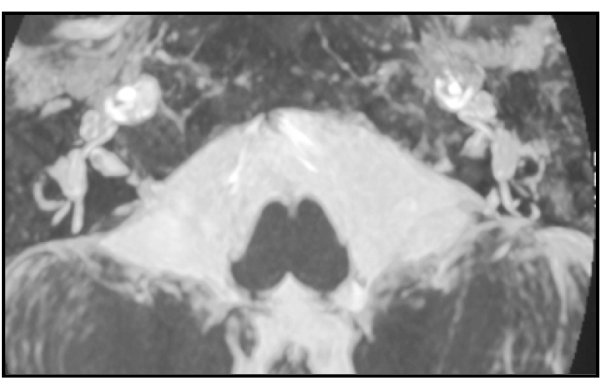

Figura 5. RNM primer caso: Sin evidencia de VIII par bilateral.

bilateral (Figura 7). La RNM de oídos muestra presencia de VIII par (Figura 8). Para evaluar funcionalidad de éste se realizó ePEAT que mostró presencia de ondas por lo que sería candidato teórico a IC (Figura 9).

\section{Caso 3}

Escolar de 10 años de edad. Con síndrome cráneofacial complejo no caracterizado e HSN profunda bilateral. Ha sido operado 2 veces de IC con falla del hardware en ambas oportunidades. Desde que falló último IC, lleva 6 años sin estimulación auditiva. El TC de oídos muestra una partición incompleta bilateral de la cóclea (Figura 10). Los ePEAT muestran una onda que se repite que pudiera corresponder a la onda V (Figura 11). Sus padres quieren tener más información antes de implantarlo por tercera vez.

\section{DISCUSIÓN}

Los estudios electrofisiológicos no reemplazan a los estudios tradicionales de evaluación auditiva ni a los

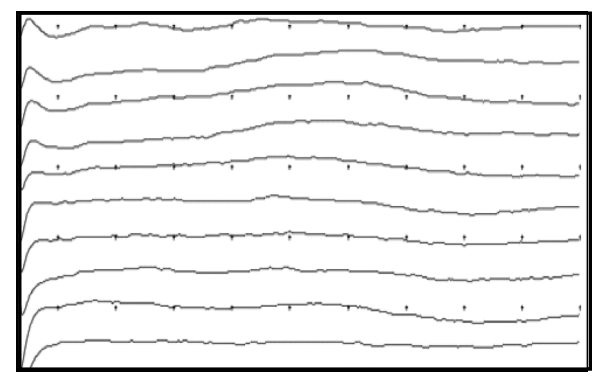

Figura 6. ePEAT primer caso: sin respuesta.

estudios por imágenes, sino que los complementan ${ }^{8-}$ ${ }^{10}$. Los casos presentados, demuestran que en casos de malformaciones de oído interno o CAl muy estrecho, en que se cuestiona seriamente la existencia de un nervio coclear funcional, y en casos de neuropatía auditiva, se hace necesario evaluar la función de la cóclea separadamente de la del nervio auditivo y la función del tronco. Para esto se utilizamos los ePEAT.

Los resultados de este examen son un factor más a considerar en la toma de decisiones para estos casos difíciles. Las condiciones desfavorables del oído medio, la edad y ausencia de estimulación auditiva son argumentos probablemente más significativos que los hallazgos aislados del ePEAT al momento de decidir la colocación de un IC, sin embargo, el ePEAT entrega información valiosísima en cuanto a si el nervio coclear será capaz de transmitir información auditiva hacia el SNC.

En pacientes con neuropatía auditiva la electrococleografía (ECochG), que permite evaluar la función de la cóclea, presenta un potencial positivo anormal (APP) probablemente asociado a la disincronía en la respuesta entre células ciliadas internas y externas. Para hacer el diagnóstico diferencial en estos pacientes en cuanto a si el 


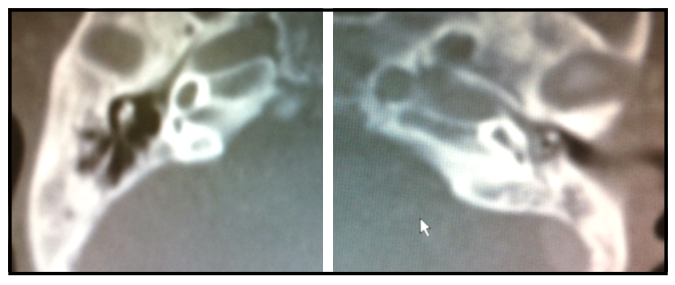

Figura 7. TC segundo caso. Partición incompleta tipo 2 a derecha y tipo 3 a izquierda. Asociado a malformación de trompa de Eustaquio y OME crónica.

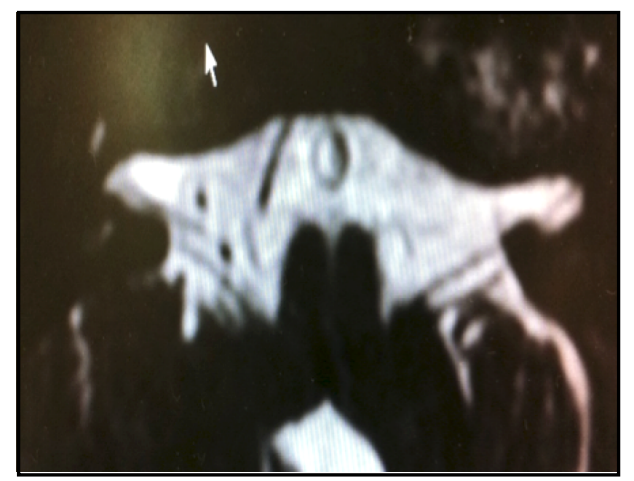

Figura 8. RNM segundo caso: se observa VIII par.

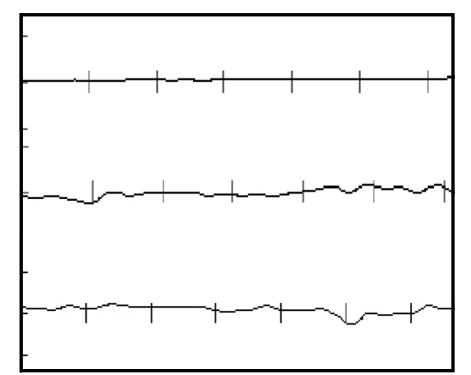

Figura 9. ePEAT segundo caso: mayor definición de onda $\mathrm{V} a$ mayor estimulación eléctrica.

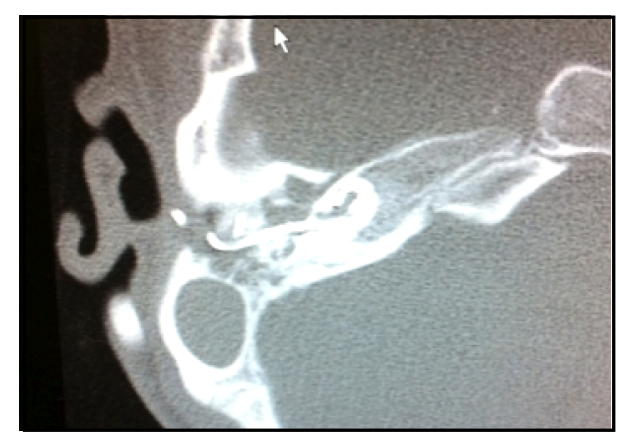

Figura 10. TC tercer caso: IC no funcionante in situ. problema viene de la cóclea 0 del nervio auditivo, se puede realizar el ePEAT que indica la función del nervio coclear y de las vías neurales al tronco.

Las curvas del ePEAT son similares al PEAT tradicional excepto en que la onda I y II aparecen con artefacto eléctrico y la onda $V$ tiene menor latencia (4 mseg) (Figura 12). En niños, el ePEAT debe realizarse en pacientes seleccionados: eCochG alterada y PEAT anormal. Cuando se observa un APP en la eCochG, PEAT anormal 0 ausente y ePEAT normal, indica que el nervio auditivo y el tronco son capaces de generar actividad neural sincrónica. Esto también significa que la lesión se encuentra en la cóclea $y / 0$ en la sinapsis inicial del nervio auditivo, constituyendo en realidad una "disincronía auditiva». Los ePEAT con ondas ausentes 0 alterados, representan una verdadera neuropatía auditiva.

El estudio de ePEAT debiera ser parte de la batería de exámenes disponibles para todos aquellos casos en que no se sabe si el implante va a servir o no.

Kileny y Zwolan reportaron 4 criterios para determinar qué pacientes debieran ser evaluados con ePEAT11:

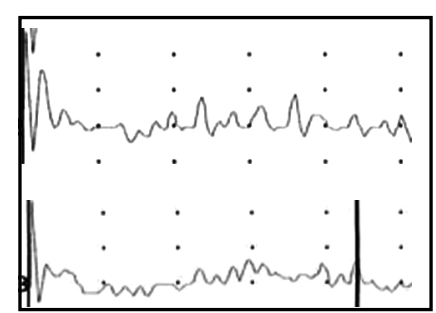

Figura 11. ePEAT tercer caso: se aprecia onda que se repite que pudiese corresponder a la onda V.

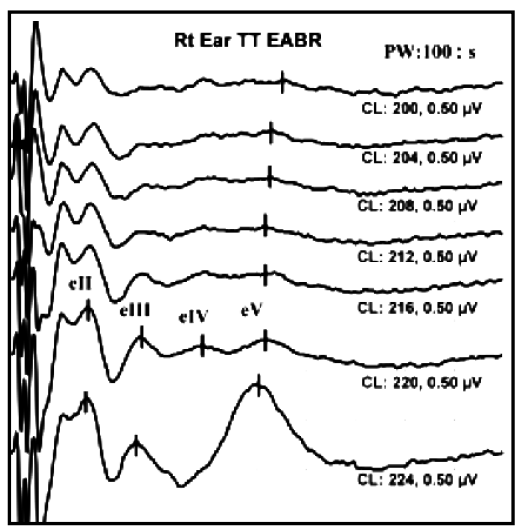

Figura 12. ePEAT: onda I y II con artefacto eléctrico. Onda V de menor latencia. 
1. Malformaciones del hueso temporal.

2. Información inconclusa sobre los umbrales auditivos en pacientes pequeños en edad cronológica o de desarrollo.

3. Pérdida auditiva profunda que excede la máxima intensidad del audiómetro.

4. Diagnóstico de neuropatía auditiva. Mediante la combinación de los estudios diagnósticos, evaluación funcional e imágenes como el TC y la RNM se puede dividir a los pacientes en cuatro categorías con predicciones generales de resultados con IC5. Éstas son:

1. HSN: patología coclear primariamente. Predicción de buenos resultados con IC.

2. Disincronía auditiva: se cree que es patología coclear primariamente. Buenos resultados predichos con IC.

3. Neuropatía auditiva: consistente con patología del nervio auditivo. Predicción de resultado subóptimo con IC.

4. Neuropatía auditiva de tronco: consistente con patología auditiva de tronco encefálico. Resultado subóptimo predicho con IC.

\section{CONCLUSIÓN}

La experiencia de nuestro grupo es inicial, los resultados pueden ser imperfectos, y las curvas obtenidas aún no son ideales, y se está trabajando en mejorar estos resultados. Sin embargo, la aplicación del ePEAT fue de gran ayuda para evaluar a los 3 pacientes presentados, nos permitió conocer las reales capacidades de ellos para transmitir un estímulo auditivo hacia el SNC, definiendo mejor las expectativas con el uso implante, asistiéndonos en nuestra toma de decisiones.

\section{BIBLIOGRAFÍA}

1. Bradley J, Beale T, Graham J, Bell M. Variable long-term outcomes from cochlear implantation in children with hypoplastic auditory nerves. Cochlear Implants Int 2008; 9(1): 34-60.

2. Acker T, Mathur NN, Savy L, Graham JM. Is there a functioning vestibulocochlear nerve? Cochlear implantation in a child with symmetrical auditory findings but asymmetric imaging. Int J Pediatr Otorhinolaryngol 2001; 57(2): 171-6.

3. Bamiou DE, Worth S, Phelps P, Sirimanna T, Rajput K. Eighth nerve aplasia and hypoplasia in cochlear implant candidates: the clinical perspective. Otol Neurotol 2001; 22(4): 492-6.

4. Gibson WP, Sanli H. Auditory neuropathy: an update. Ear Hear 2007; 28(2 Suppl): 102S-6S.

5. Gardner-Berry K, Gibson WP, Sanli H. Pre-operative testing of patients with neuropathy or dys-synchrony. The Hearing Journal 2005; 58(11): 24-31.

6. Kileny PR, Zwolan TA, Zimmerman-Phillips S, Telian SA. Electrically evoked auditory brain-stem response in pediatric patients with cochlear implants. Arch Otolaryngol Head Neck Surg 1994; 120(10): 1083-90.

7. Kileny PR, Zwolan TA, Zimmerman-Phillips S, Kemink JL. A comparison of round-window and transtympanic promontory electric stimulation in cochlear implant candidates. Ear Hear 1992; 13(5): 294-9.

8. KILENY PR. Use of electrophysiologic measures in the management of children with cochlear implants: brainstem, middle latency, and cognitive (P300) responses. Am J Otol 1991; 12 Suppl: 37-42; discussion 3-7.

9. ShaLlop JK. Objective electrophysiological measures from cochlear implant patients. Ear Hear 1993; 14(1): 58-63.

10. Brown CJ, Abbas PJ, Fryauf-Bertschy H, Kelsay D, Gantz BJ. Intraoperative and postoperative electrically evoked auditory brain stem responses in nucleus cochlear implant users: implications for the fitting process. Ear Hear 1994; 15(2): 168-76.

11. Kileny PR, Zwolan TA. Pre-perioperative, transtympanic electrically evoked auditory brainstem response in children. Int $J$ Audiol 2004; 43 Suppl 1: S16-21. 